\title{
Harmonization of adverse events monitoring following thoracic surgery: Pursuit of a common language and methodology
}

\author{
Gregory Sigler, MDCM, ${ }^{\mathrm{a}, \mathrm{b}}$ Caitlin Anstee, BA, ${ }^{\mathrm{b}, \mathrm{c}}$ and Andrew J. E. Seely, MD, PhD, FRCSC ${ }^{\mathrm{b}, \mathrm{c}}$
}

\section{ABSTRACT}

Objective: Thoracic surgery carries significant risk of postoperative adverse events (AEs). Multiple international recording systems are used to define and collect AEs following thoracic surgery procedures. We hypothesized that a simple-yetubiquitous approach to AE documentation could be developed to allow universal data entry into separate international databases.

Methods: AE definitions of the Canadian Association of Thoracic Surgeons (CATS) system and 4 international databases were matched and compared. This consisted of reviewing the definition of each $A E$ as described by their respective database and assessing compatibility with the CATS system. We developed a single set of 4 dropdown menus to enable clear classification and facilitated data entry, using 3 singleselect mandatory lists and 1 multiselect optional list classifying type and severity of these events.

Results: The CATS data elements were harmonized (ie, perfect or good) with $100 \%$ (European Society of Thoracic Surgeons), 89\% (Society of Thoracic Surgeons), 74\% (Esophagectomy Complications Consensus Group), and 73\% (National Surgical Quality Improvement Program) of respective data elements. The addition of 17 AEs and 2 complication modifiers to the CATS system was implemented to achieve complete harmonization. Consequently, $100 \%$ of $\mathrm{AE}$ data elements currently included in all 4 international databases are perfectly or wellharmonized with the revised 4-choice drop down menu.

Conclusions: We describe a framework for a ubiquitously applicable approach to AE monitoring following thoracic surgery harmonized with AE definitions of all major thoracic international associations. Use of this AE collection framework allows for comprehensive evaluation of both the incidence and severity of all AEs after thoracic surgery along with quality indicators. (JTCVS Open 2021;6:250-6)

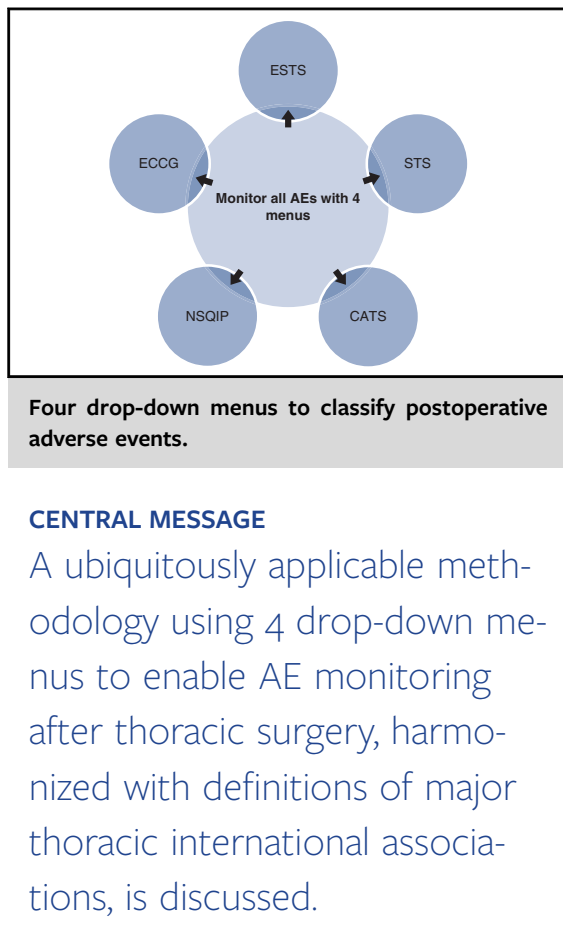

\section{PERSPECTIVE}

Multiple international recording systems are used to define and collect AEs following thoracic surgery procedures. A simple, ubiquitous approach to $A E$ documentation was developed to allow universal data entry into separate international databases. This framework allows for comprehensive evaluation of both incidence and severity of all AEs after thoracic surgery, with potential for broad application.

See Commentary on page 257 .
From the Divisions of a General Surgery and ${ }^{\mathrm{c}}$ Thoracic Surgery, Department of Surgery, and ${ }^{\mathrm{b}}$ Ottawa Hospital Research Institute, University of Ottawa, Ottawa, Ontario, Canada.

Received for publication March 29, 2021; accepted for publication March 29, 2021; available ahead of print April 29, 2021.

Address for reprints: Andrew J. E. Seely, MD, PhD, FRCSC, 501 Smyth Rd, Box 708, Ottawa, Ontario, Canada, K1H 8L6 (E-mail: aseely@ohri.ca).

2666-2736

Copyright (C) 2021 The Author(s). Published by Elsevier Inc. on behalf of The American Association for Thoracic Surgery. This is an open access article under the CC BY license (http://creativecommons.org/licenses/by/4.0/).

https://doi.org/10.1016/j.xjon.2021.03.021

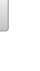




\section{Abbreviations and Acronyms \\ $\mathrm{AE}=$ adverse event \\ CATS $=$ Canadian Association of Thoracic Surgeons \\ ECCG $=$ Esophagectomy Complications Consensus Group \\ ESTS = European Society of Thoracic Surgeons \\ NSQIP = National Surgical Quality Improvement Program \\ STS = Society of Thoracic Surgeons \\ TM\&M $=$ Thoracic Morbidity and Mortality}

patient experience, ${ }^{10}$ and increases health care costs. ${ }^{5,11}$ In Canada, treatments for individual AEs range from $\$ 4000$ to $\$ 12,000 .{ }^{12}$ While some AEs inevitably occur, between $37 \%$ and $51 \%$ of reported AEs are potentially preventable and cost the Canadian health care system \$397 million/ year. ${ }^{7,12}$ Comprehensive monitoring and documentation of AEs is therefore essential to inform clinical research and quality-improvement programs.

Multiple international recording systems are used to define and collect AEs following thoracic surgery procedures. These include the Society of Thoracic Surgeons (STS), ${ }^{13}$ the European Society of Thoracic Surgeons (ESTS), ${ }^{14}$ the Esophagectomy Complications Consensus Group (ECCG), ${ }^{15}$ the National Surgical Quality Improvement Program (NSQIP), ${ }^{16}$ and the Thoracic Morbidity and Mortality (TM\&M) classification system ${ }^{1}$ adopted by the Canadian Association of Thoracic Surgeons (CATS). Increasingly, documentation of both severity and incidence of AEs has demonstrated enhanced capacity to track the impact of an $\mathrm{AE}$ in addition to its occurrence. The Clavien-Dindo classification system is a broadly applicable and validated tool used to track both incidence and severity of surgical complications based on the degree of therapeutic intervention required to treat the AE. ${ }^{17,18}$ Within thoracic surgery, the TM\&M system is a standardized approach to identify both severity and incidence of thoracic-related postoperative AEs based on the Clavien-Dindo model. ${ }^{1}$ The TM\&M model (https://www.ottawatmm.org) has demonstrated feasibility, reliability, and reproducibility as a tool for systematic monitoring, reporting, and evaluation of postoperative complications following thoracic surgery. ${ }^{19}$ It has been adopted by surgical groups internationally to gather prospective data regarding the burden of surgical complications and thus allows for continuous quality assessment. ${ }^{20,21}$ As it stands, there exists variation across systems in both which AEs are recorded and what criteria are used to describe these events. Although ESTS and STS have harmonized their AE definitions, ${ }^{14}$ they do not systematically measure AE incidence and severity. Substantial differences between NSQIP and TM\&M data-collection systems exist, which call for improved harmonization, as well as inclusion of relevant thoracic-specific $\mathrm{AE}$ within the NSQIP system. ${ }^{2}$ This discordance limits the capacity to share information between databases. In addition, if a center wishes to participate in 2 international databases, it must collect both data separately. Improving capacity to simply record all AEs in a manner that it could be used for any international database would benefit the international research community interested in $\mathrm{AE}$ research and quality improvement.

We hypothesized that a simple-yet-ubiquitous approach to AE documentation could be developed that would allow universal data entry into the separate international databases. In other words, we sought to create a single AEdocumentation system that could feed data to all systems, including STS, ESTS, TM\&M, ECCG, and NSQIP. In this short paper, we introduce this system and formally evaluate whether this AE documentation and classification tool would enable ubiquitous and effective translation into other validated international $\mathrm{AE}$ classification systems. Our intention was to use this as a method to improve deficiencies in our own system, which we ultimately hope will allow improved collaboration among international research societies.

\section{METHODS}

We aimed to create an AE classification system capable of harmonizing with all other database definitions. We developed a single set of 4 dropdown menus to enable clear classification and facilitated data entry (Figure 1). This system is labeled as the CATS system, as it has been adopted by that organization. The approach uses 3 single-select lists and 1 optional multiselect list to track the type and severity of these events. The single-select options include system (eg, pleural, pulmonary, gastrointestinal, neurologic, etc), type of complication (eg, pneumonia, air leak, etc), and Clavien-Dindo grade (eg, Grade I to V). Finally, the multiselect list includes modifiers (eg, resulted in prolonged length of stay, required emergency department visit, or hospital readmission, etc) that can be associated with each $\mathrm{AE}$.

\section{AE Definitions}

The AE definitions of the CATS system and those of ESTS, STS, ECCG, and NSQIP databases were matched and compared. The matching process consisted of reviewing the definition of each $\mathrm{AE}$ as described by their respective database and assessing compatibility with those defined by the CATS system (Figure 2). Any discrepancies between definitions and grades of AEs were explicitly noted. Tables were created to facilitate systemsbased comparison (see Online Data Supplement 1). The degree to which AE data elements of the classification systems were harmonized with the CATS system were categorized as Perfect (ie, exact wording), Good (ie, nearly exact wording), or Non-Harmonized. The non-harmonized definitions were compiled and used to provide recommendations for prospective modifications to the CATS system (see Online Data Supplement 2).

\section{AE Collection}

The methods of AE data collection not only require clear definitions that are harmonized across international groups but also necessitate prospective recording of $\mathrm{AE}$ on a daily basis, ensuring the treatment team is participating in the recording of AEs. AEs may be recorded using a set of 4 


\section{Harmonization of Adverse Event Monitoring in Thoracic Surgery}
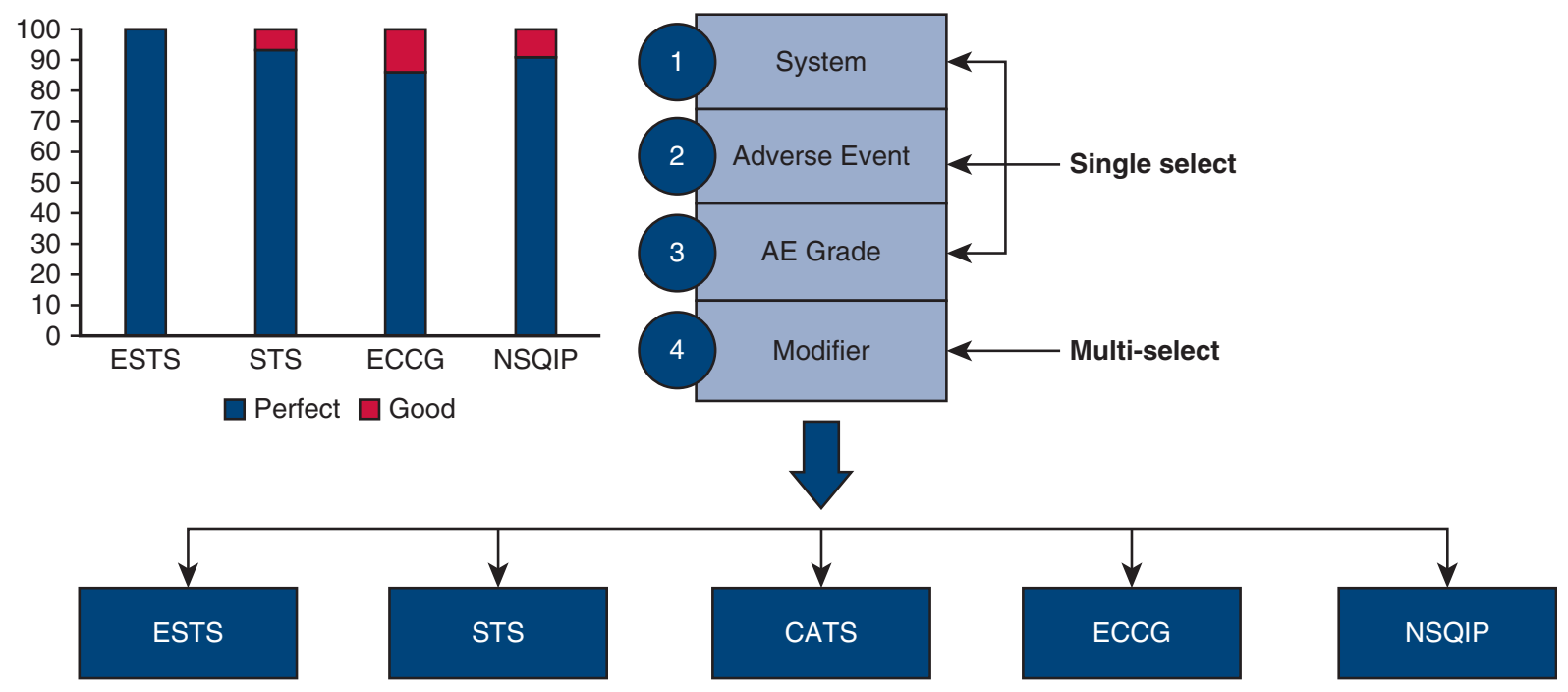

Ubiquitous methodology for adverse event monitoring

$\mathrm{AE}=$ adverse event; ESTS = European Society of Thoracic Surgeons; STS = Society of Thoracic Surgeons; CATS = Canadian Association of Thoracic Surgeons; ECCG = Esophagectomy Complications Consensus Group; NSQIP = National Surgical Quality Improvement Program

FIGURE 1. Our harmonized system uses a single set of 4 drop-down menus to classify postoperative adverse events ( $A E s$ ), including system, adverse event, grade, and modifier. AEs may be fed into all international systems including the Canadian Association of Thoracic Surgeons (CATS), Society of Thoracic Surgeons (STS), European Society of Thoracic Surgeons (ESTS), Esophagectomy Complications Consensus Group (ECCG), and National Surgical Quality Improvement Program (NSQIP).

drop down menus (3 single-select and 1 multiselect). In addition to daily prospective entry, weekly review is recommended to highlight all AEs and their incidence and severity, ensuring multidisciplinary discussion regarding the labeling of AEs in both incidence and severity. This prospective daily entry and weekly discussion is recommended as optimal to capture all AEs after all surgeries at any institution.

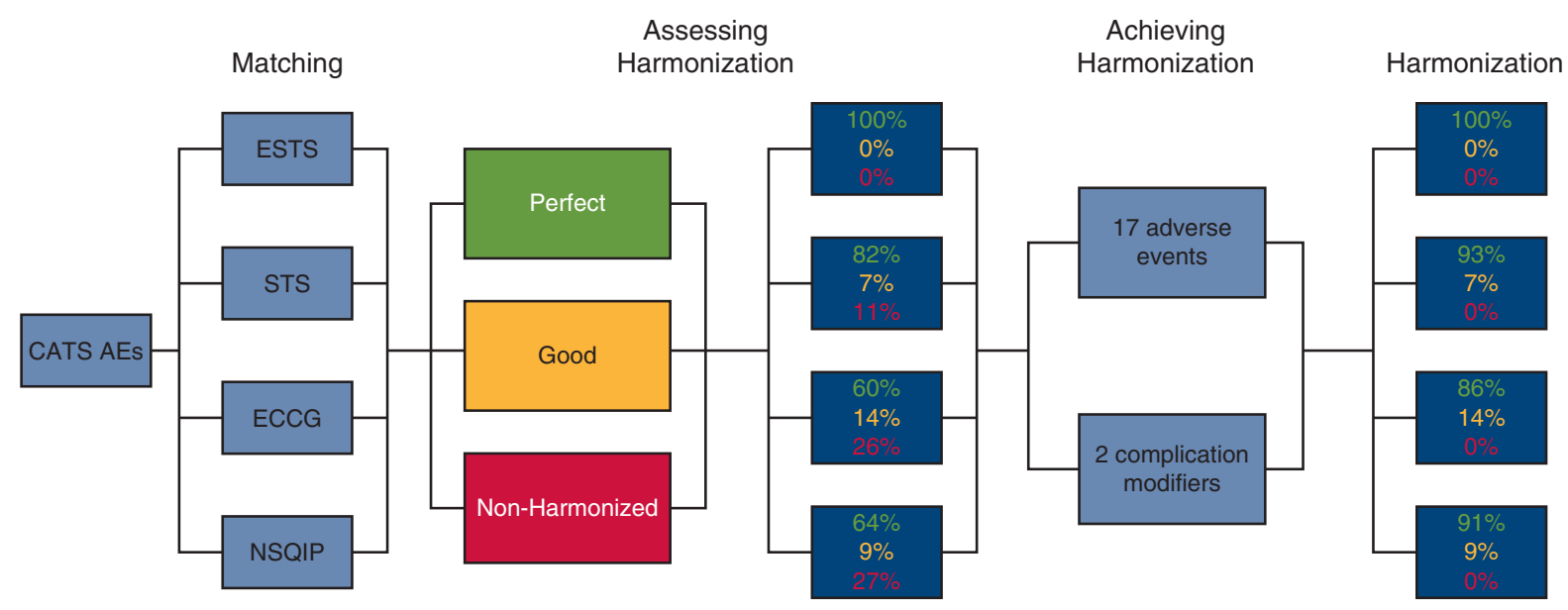

FIGURE 2. Flow diagram of the matching and harmonization process, read from left to right. The "Matching" process consisted of reviewing the definition of each adverse event $(A E)$ as described by their respective database and assessing compatibility with those defined by the Canadian Association of Thoracic Surgeons' (CATS) system. “Assessing Harmonization” was accomplished by categorizing as Perfect (ie, exact wording), Good (ie, nearly exact wording), or Non-Harmonized the AE data elements of each classification system. The non-harmonized definitions were compiled and used to provide recommendations for prospective modifications to the CATS system ("Achieving Harmonization"), including the addition of 17 AEs and 2 complication modifiers. Following these modifications, $100 \%$ of AE data elements currently included in all international databases are perfectly or well harmonized with the revised four-drop down menu ("Harmonization"). NSQIP, National Surgical Quality Improvement Program; ECCG, Esophagectomy Complications Consensus Group; STS, Society of Thoracic Surgeons; ESTS, European Society of Thoracic Surgeons. 
Total Number of AEs Defined

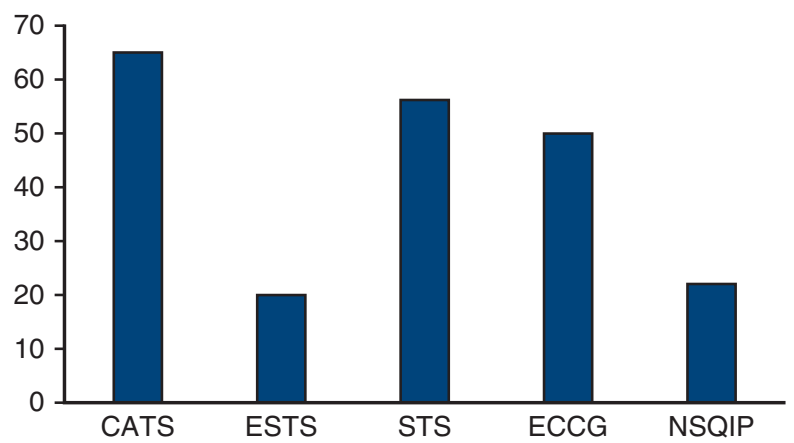

FIGURE 3. The number of postoperative adverse events (AEs) defined by each classification system: Canadian Association of Thoracic Surgeons (CATS), 65; European Society of Thoracic Surgeons (ESTS), 20; Society of Thoracic Surgeons (STS), 56; Esophagectomy Complications Consensus Group $(E C C G)$, 50; National Surgical Quality Improvement Program (NSQIP), 22.

\section{RESULTS}

The total number of AEs defined in the CATS, ESTS, STS, ECCG, and NSQIP classification systems was 65, 20, 56, 50, and 22, respectively (Figure 3). The CATS data elements were harmonized (ie, perfect or good) with $100 \%, 89 \%, 74 \%$, and $73 \%$ for ESTS, STS, ECCG, and NSQIP, respectively (Figure 4). Additional AEs from other classification systems that CATS had not defined were identified. To achieve complete harmonization, the following changes to the CATS system were proposed: the addition

\section{Percent Harmonization (CATS to Other)}

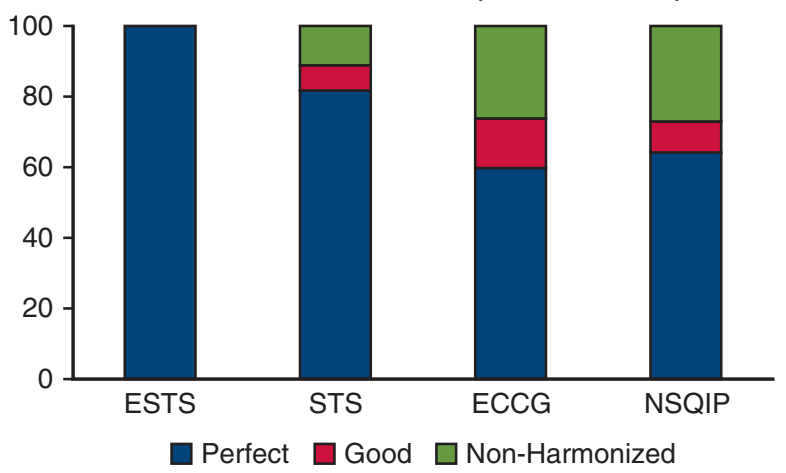

FIGURE 4. The initial matching process of the Canadian Association of Thoracic Surgeons (CATS) data elements with those of the European Society of Thoracic Surgeons (ESTS), Society of Thoracic Surgeons (STS), Esophagectomy Complications Consensus Group (ECCG), and National Surgical Quality Improvement Program (NSQIP). These were harmonized (ie, perfect or good) with $100 \%, 89 \%, 74 \%$, and $73 \%$ for ESTS, STS, ECCG, and NSQIP, respectively. The $11 \%, 26 \%$, and $27 \%$ of nonharmonized data elements for STS, ECCG, and NSQIP, respectively, represent adverse events not previously defined by the CATS system.
TABLE 1. Proposed changes to the Canadian Association of Thoracic Surgeons (CATS) system to achieve complete harmonization with all other adverse event classification systems

\begin{tabular}{ll}
\hline \multicolumn{1}{c}{ System } & \multicolumn{1}{c}{ Adverse event } \\
\hline Cardiovascular & Cardiac arrest requiring CPR \\
Gastrointestinal & C-Diff \\
\hline Gastrointestinal & Feeding J-tube complication \\
Gastrointestinal & Liver dysfunction \\
\hline Gastrointestinal & Pancreatitis \\
\hline Gastrointestinal & Pyloromyotomy/pyloroplasty complication \\
\hline Gastrointestinal & Small bowel obstruction \\
\hline Gastrointestinal & Stricture \\
\hline Gastrointestinal & Intra-abdominal bleeding \\
\hline Gastrointestinal & Acute diaphragmatic hernia \\
\hline Gastrointestinal & Intra-abdominal abscess \\
\hline Other & Anemia or anemia requiring transfusion \\
\hline Other & Bacteremia or bacteremic shock \\
\hline Other & Central-line infection \\
\hline Neurologic & Delirium tremens \\
\hline Pulmonary & Acute aspiration \\
\hline Pulmonary & Pulmonary abscess \\
\hline Complication modifiers & Discharged with chest tube \\
\hline Discharged with home O2 \\
\hline
\end{tabular}

A total of 17 adverse events and 2 complication modifiers were added. These include adverse events in multiple systems-cardiovascular (1), gastrointestinal (10), other (3), neurologic (1), and pulmonary (2) —and complication modifiers such as "discharged with chest tube" and "discharged with home O2." $C P R$, Cardiopulmonary resuscitation; C-Diff, Clostridium difficile.

of 17 AEs and 2 complication modifiers (Table 1). These include AEs in multiple systems-cardiovascular (1), gastrointestinal (10), neurologic (1), other (3), and pulmonary (2) — and complication modifiers such as "Discharged with chest tube" and "discharged with home O2." These additions resulted in the CATS TM\&M system being perfectly harmonized with $100 \%, 93 \%, 86 \%$, and $91 \%$ of ESTS, STS, ECCG, and NSQIP data elements, respectively, with the remainder being well harmonized (Figure 5). The remaining terms that were not perfectly harmonized are those that were well-harmonized during the initial matching process, whose definitions could not be altered. In summary, $100 \%$ of $\mathrm{AE}$ data elements currently included in all 4 international databases are perfectly or well harmonized with the revised 4-drop down menu.

The recommendations for changes to the CATS classification tool were presented at the 2019 CATS National Database Meeting in April 2019. These recommendations were discussed, refined, and subsequently adopted by the CATS Database Governance Committee. Participants of the CATS national database began collecting data using these additional definitions as of July 2019. 


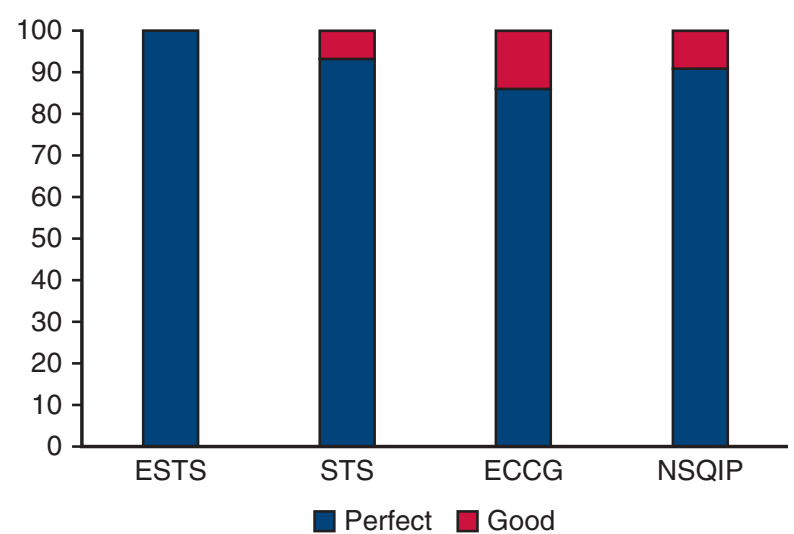

FIGURE 5. Following the addition of 17 postoperative adverse events and 2 complication modifiers to the Canadian Association of Thoracic Surgeons (CATS) system, it was perfectly harmonized with $100 \%, 93 \%$, $86 \%$, and $91 \%$ of the European Society of Thoracic Surgeons (ESTS), Society of Thoracic Surgeons (STS), Esophagectomy Complications Consensus Group (ECCG), and National Surgical Quality Improvement Program (NSQIP) data elements, respectively, with the remainder being well harmonized. The remaining terms that were not perfectly harmonized are those that were well-harmonized during the initial matching process, whose definitions could not be altered.

\section{DISCUSSION}

Our comparison provides insight into the degree of similarities and differences between the AE monitoring systems studied. We developed a ubiquitous AE classification system with 4 drop-down menus and matched them with the other international databases. The ESTS tracks 20 AEs and matched $100 \%$ (20/20), which can be attributed to previous collaboration between the 2 societies. ${ }^{10}$ The STS definitions were considered $89 \%(50 / 56)$ perfect or good harmonization. This database identified 2 new AEs and 2 new complication modifiers (see Online Data Supplement 2). The ECCG definitions were far less harmonized at 74\% (37/ 50), although this database identified 13 AEs now included in the CATS system (see Online Data Supplement 2). The NSQIP system did not uniquely identify any additional terms and was matched to $73 \%(16 / 22)$. There are 1,1 , and 2 instances in which 2 of 3 of these databases-STS, ECCG, and NSQIP, respectively - both identified a term not previously monitored by the TM\&M system. There are 2 additional AEs that are monitored by all 3 of these databases, which have now been incorporated into the CATS system, which now include Clostridium difficile infection and bacteremia or bacteremic shock (sepsis, septic shock) as independent AEs. Thus, following the addition of these AEs, all AE recorded were well or perfectly harmonized with the STS, ESTS, ECCG, and NSQIP AE definitions. This capacity to create a ubiquitous data entry method in general demonstrates the broad applicability of the Clavien-Dindo classification schema adopted by TM\&M and CATS. The utility of this ubiquitously applicable 4-drop down menu is that any vendor can see this information and thus feed data to ESTS, STS, CATS, NSQIP, and ECCG as they see fit. It can be adopted by any software tool and allow databases to be analyzed in a more synchronous manner.

Interestingly, our initial comparison of the databases showed that there were only 6 AEs (myocardial infarction, pulmonary embolism, cerebrovascular complication, pneumonia, acute renal failure, wound infection) and 1 complication modifier (requiring reintubation) that are universally defined by all 5 databases. A comparison of only CATS, ESTS, STS, and ECCG increases the total of universally defined AEs to 13, with the addition of atrial arrythmia, ventricular arrythmia, delirium, recurrent nerve palsy, chylothorax, atelectasis, and acute respiratory distress syndrome (Online Data Supplement 1). This further highlights the potential benefits of our model to the international research community interested in $\mathrm{AE}$ research and quality improvement. We have proposed a single AE documentation system that could feed data to all systems, including CATS, ESTS, STS, ECCG and NSQIP. The modified CATS system has become universally applicable to its four international counterparts. Our simple online tool allows the user to easily input data on 82 AEs and 10 complications modifiers in a straightforward and efficient manner. It holds an advantage over traditional methods of monitoring AEs in that it is an easily-accessible web-based platform that can be used by multiple stakeholders simultaneously and eliminates the need to fill out a lengthy case report form. While we do not currently have any formal evaluation of the time it would take to enter data, it would practically seem more efficient.

It is always a work in progress to review and maintain clearly defined AEs after thoracic surgery. One limitation in this work was the fact some AE definitions are not explicitly defined, which made determining harmonization challenging. As a small example, "deep vein thrombosis" is classified by ECCG as an AE; however, no specific criteria are provided to define this. In such cases, we considered the definition perfectly harmonized. This may have led to an overestimation in the degree of real-life harmonization if the CATS system with other databases.

The primary author (G.S.) performed a review of the data elements of each classification system. The elements whose definitions either matched exactly or whose meanings were clinically indistinguishable were considered be in "Perfect" harmonization. Any definition whose wording did not match exactly was reviewed with the supervising author (A.S.) to determine its degree of harmonization. The authors recognize that this process represents a limitation in our methodology, as our outcomes are based on 2 authors' opinions as opposed to a more extensive and rigorous evaluation of opinions of other surgeons and experts in the field. However, we feel that the definitions of each classification system as a whole are straightforward and intentionally highly objective. 


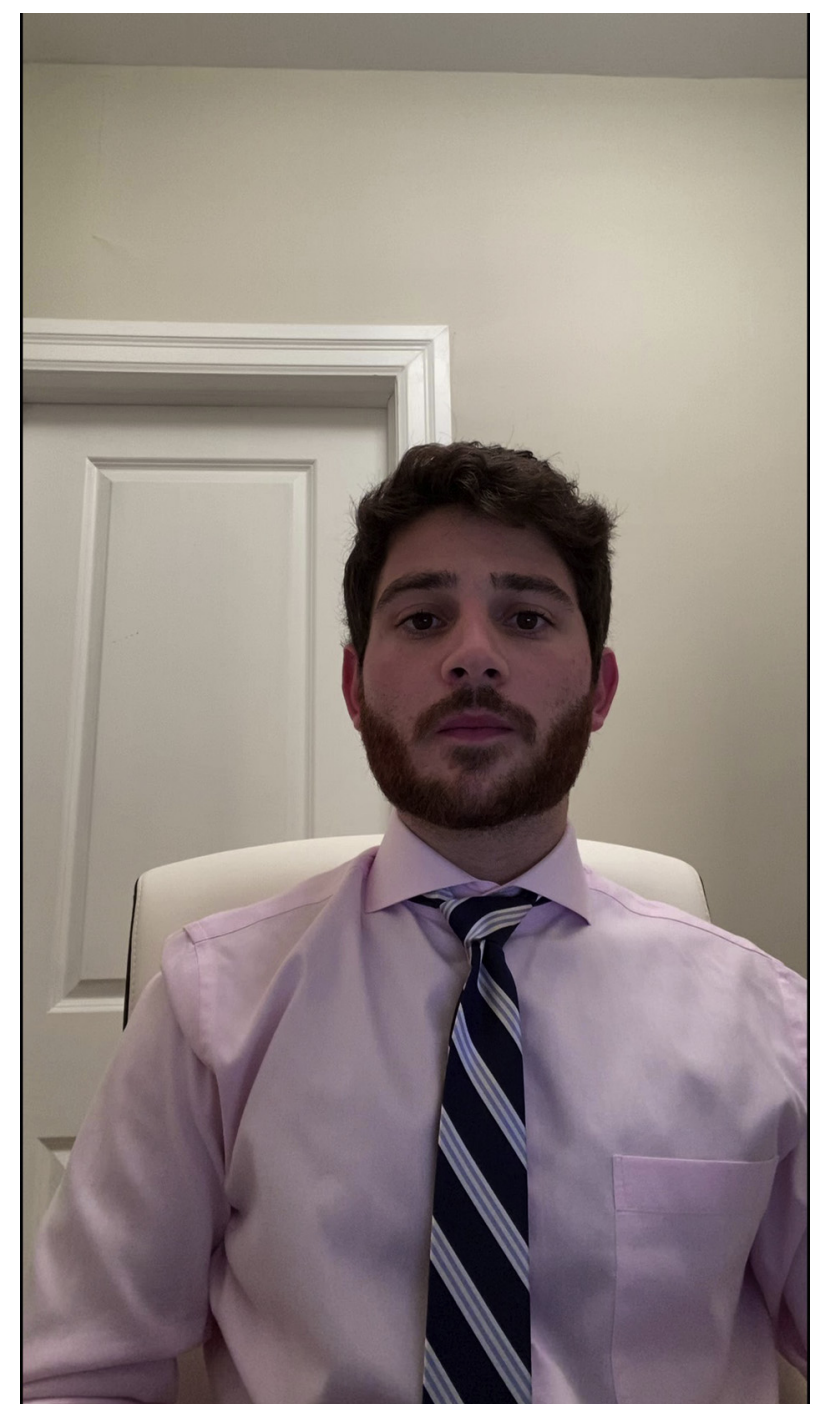

VIDEO 1. Video recording of primary author (Gregory Sigler) describing the purpose, methodology, outcomes and implications of the project. Video available at: https://www.jtcvs.org/article/S2666-2736(21)00078-4/fulltext.

The implementation of a ubiquitously applicable AE classification tool offers the ability to compare and share data with other international AE-monitoring systems. Broadening the volume of data available to assess surgical quality improvement has vast applications, including the study of more rare pathologies, procedures, and complications. The TM\&M website reflects these changes (https:// www.ottawatmm.org) and provides a tool to facilitate translation of corresponding data elements between classification systems. While our project draws a specific focus to AEs following thoracic surgery, we believe our work employs a simple strategy, which can be applied broadly to monitoring AEs following many types of surgical procedures. The goal of harmonization of data collection is not synonymous with joining together multiple databases. It is rather to ensure that AEs are simply collected in the same manner, capable of participating in any or all international data-collection systems.

\section{CONCLUSIONS}

This paper provides a framework for discussion and advancement toward a harmonized approach for AE definition and recording following thoracic surgery (Video 1). We hope that our finding that a single set of 4 drop-down menus that can enable universal $\mathrm{AE}$ data collection is useful to surgeons and societies interested in broad international benchmarking. If $\mathrm{AE}$ data could be pooled across international databases, there will be greater to capacity to both understand the incidence and impact of AEs and enhance research and quality improvement programs to reduce or mitigate AEs.

\section{Conflict of Interest Statement}

The authors reported no conflicts of interest.

The Journal policy requires editors and reviewers to disclose conflicts of interest and to decline handling or reviewing manuscripts for which they may have a conflict of interest. The editors and reviewers of this article have no conflicts of interest.

We acknowledge all the thoracic surgery residents and staff who have contributed to adverse event collection on a day-to-day, week-to-week basis.

\section{References}

1. Seely AJE, Ivanovic J, Threader J, Al-Hussaini A, Al-Shebab D, Ramsay T, et al Systematic classification of morbidity and mortality after thoracic surgery. Ann Thorac Surg. 2010;90:936-42.

2. Ivanovic J, Seely AJE, Anstee C, Villeneuve PJ, Gilbert S, Maziak DE, et al. Measuring surgical quality: comparison of postoperative adverse events with the American College of Surgeons NSQIP and the thoracic morbidity and mortality classification system. J Am Coll Surg. 2014;218:1024-31.

3. Andalib A, Ramana-Kumar AV, Bartlett G, Franco EL, Ferri LE. Influence of postoperative infectious complications on long-term survival of lung cancer patients: a population-based cohort study. J Thorac Oncol. 2013; 8:554-61.

4. Khuri SF, Henderson WG, DePalma RG, Mosca C, Healey NA, Kumbhani DJ, et al. Determinants of long-term survival after major surgery and the adverse effect of postoperative complications. Ann Surg. 2005;242:326-41.

5. Khan NA, Quan H, Bugar JM, Lemaire JB, Brant R, Ghali WA, et al. Association of postoperative complications with hospital costs and length of stay in a tertiary care center. J Gen Intern Med. 2006;21:177-80.

6. Irshad K, Feldman LS, Chu VF, Dorval JF, Baslaim G, Morin JE, et al. Causes of increased length of hospitalization on a general thoracic surgery service: a prospective observational study. Can J Surg. 2002;45:264-8.

7. Baker GR, Norton PG, Flintoft V, Blais R, Brown A, Cox J, et al. The Canadian adverse events study: the incidence of adverse events among hospital patients in Canada. CMAJ. 2004; 170:1678-86.

8. Brown LM, Thibault DP, Kosinski AS, Cooke DT, Onaitis MW, Gaissert HA, et al. Readmission after lobectomy for lung cancer: not all complications contribute equally. Ann Surg. August 28, 2019 [Epub ahead of print].

9. Dickinson KJ, Taswell JB, Allen MS, Blackmon SH, Nichols FC, Shen R, et al. Unplanned readmission after lung resection: complete follow-up in a 1-year cohort with identification of associated risk factors. Ann Thorac Surg. 2017; 103:1084-91.

10. Harrison R, Walton M, Manias E, Smith-Merry J, Kelly P, Iedema R, et al. The missing evidence: a systematic review of patients' experiences of adverse events in health care. Int J Qual Health Care. 2015;27:424-42. 
11. Vonlanthen R, Slankamenac K, Breitenstein S, Puhan MA, Muller MK, Hahnloser D, et al. The impact of complications on costs of major surgical procedures: a cost analysis of 1200 patients. Ann Surg. 2011;254:907-13.

12. Canadian Patient Safety Institute. The Economics of Patient Safety in Acute Care: Technical Report. Ottawa: Canadian Patient Safety Institute; 2012.

13. The Society of Thoracic Surgeons General Thoracic Surgery database analyzed procedure data collection form, Version $2.41 ; 2018$. Available at: https://www. sts.org/registries-research-center/sts-national-database/general-thoracic-surgerydatabase/data-collection. Accessed March 27, 2019.

14. Fernandez FG, Falcoz PE, Kozower BD, Salati M, Wright CD, Brunelli A. The Society of Thoracic Surgeons and the European Society of Thoracic Surgeons General Thoracic Surgery databases: joint standardization of variable definitions and terminology. Ann Thorac Surg. 2015;99:368-76.

15. Low DE, Alderson D, Cecconello I, Chang AC, Darling GE, D'Journo XB, et al. International consensus on standardization of data collection for complications associated with esophagectomy: Esophagectomy Complications Consensus Group (ECCG). Ann Surg. 2015;262:286-94.

16. User Guide for the 2017 ACS NSQIP Participant Use Data File (2018). American College of Surgeons. National Surgical Quality Improvement Program. Available at: https://www.facs.org/quality-programs/acs-nsqip/participant-use. Accessed March 27, 2019.
17. Clavien PA, Sanabria JR, Strasberg SM. Proposed classification of complications of surgery with examples of utility in cholecystectomy. Surgery. 1992;111: 518-26.

18. Dindo D, Demartines N, Clavien PA. Classification of surgical complications: a new proposal with evaluation in a cohort of 6336 patients and results of a survey. Ann Surg. 2004;240:205-13.

19. Ivanovic J, Threader J, Alhussaini A, Al-Shebab D, Ramsay T, Gilbert S, et al. Testing the reliability and reproducibility of the Ottawa Hospital thoracic morbidity and mortality classification system. Ann Thorac Surg. 2011;91:387-93.

20. Salati M, Pompili C, Refai M, Xiumè F, Sabbatini A, Brunelli A, et al. The use of the thoracic morbidity and mortality system for the internal analysis of performance: a case-matched temporal audit. Eur J Cardiothorac Surg. 2014;45: 859-63.

21. Salati M, Refai M, Pompili C, Xiumè F, Sabbatini A, Brunelli A, et al. Major morbidity after lung resection: a comparison between the European Society of Thoracic Surgeons database system and the thoracic morbidity and mortality system. J Thorac Dis. 2013;5:217-22.

Key Words: thoracic surgery, adverse event monitoring, harmonization, Clavien-Dindo model 